\title{
Synthesis and Structural Characterization of A Series of Lanthanide(II) Amides: Steric Effect of Amido Ligand
}

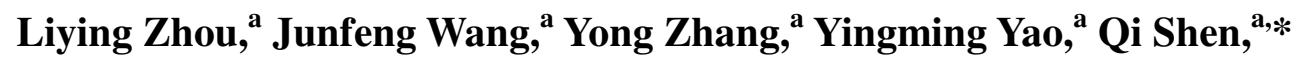

${ }^{a}$ Key Laboratory of Organic Synthesis, Jiangsu Province, Department of Chemistry and Chemical Engineering, Suzhou University, Suzhou, 215123, China.

Table S1. Details of the crystallographic data of complex 4

\begin{tabular}{ll|ll}
\hline \hline Empirical formula & $\mathrm{C}_{49} \mathrm{H}_{84} \mathrm{Cl}_{2} \mathrm{LiN}_{2} \mathrm{O}_{3} \mathrm{Si}_{2} \mathrm{Yb}$ & $\gamma\left({ }^{\circ}\right)$ & 90 \\
Formula weight & 1056.24 & $V / \AA^{3}$ & $11229.5(15)$ \\
Crystal colour & orange & $Z$ & 8 \\
Temperature / K & $223(2)$ & $D_{\text {calc }} / \mathrm{mg} \mathrm{m}^{-3}$ & 1.250 \\
Wavelength / $\AA$ & 0.71070 & Absorption & 1.840 \\
Size (mm) & $0.60 \times 0.58 \times 0.50$ & $F(000)$ & 4408 \\
Crystal system & orthorhombic & $\theta /{ }^{\circ}$ & $3.04-25.35$ \\
Space group & $\mathrm{Pb} \mathrm{c} \mathrm{a}$ & Reflection collected & 101353 \\
$a(\AA)$ & $16.4127(13)$ & Independent reflections & 10254 \\
$b\left(^{-1}\right)$ & $18.1102(14)$ & Parameters & 545 \\
$c(\AA)$ & $37.780(3)$ & $R[I>2 \sigma(I)]$ & 0.1151 \\
$\alpha\left(^{\circ}\right)$ & 90 & $w R$ & 0.1855 \\
$\beta\left(^{\circ}\right)$ & 90 & GOF on $F^{2}$ & 1.161 \\
\hline \hline
\end{tabular}


Table S2. Selected bond distances $(\AA)$ and angles $\left(^{\circ}\right)$ for $\mathbf{4}$

\begin{tabular}{|c|c|c|c|}
\hline Bond & Dist. $(\AA)$ & Bond & Dist. $(\AA)$ \\
\hline $\mathrm{Yb}(1)-\mathrm{N}(1)$ & $2.171(9)$ & $\operatorname{Si}(1)-N(1)$ & $1.697(10)$ \\
\hline $\mathrm{Yb}(1)-\mathrm{N}(2)$ & $2.175(9)$ & $\operatorname{Si}(2)-N(2)$ & $1.710(8)$ \\
\hline $\mathrm{Yb}(1)-\mathrm{Cl}(2)$ & $2.479(3)$ & $\mathrm{O}(1)-\mathrm{Li}(1)$ & $1.95(3)$ \\
\hline $\mathrm{Yb}(1)-\mathrm{Cl}(1)$ & $2.516(3)$ & $\mathrm{O}(2)-\mathrm{Li}(1)$ & $1.86(3)$ \\
\hline $\mathrm{Cl}(1)-\operatorname{Li}(1)$ & $2.38(2)$ & $\mathrm{O}(4)-\mathrm{Li}(1)$ & $1.89(2)$ \\
\hline Angle & $\left({ }^{\circ}\right)$ & Angle & $\left({ }^{\circ}\right)$ \\
\hline $\mathrm{N}(1)-\mathrm{Yb}(1)-\mathrm{N}(2)$ & 119.1(3) & $\mathrm{O}(2)-\mathrm{Li}(1)-\mathrm{O}(4)$ & $108.1(13)$ \\
\hline $\mathrm{N}(1)-\mathrm{Yb}(1)-\mathrm{Cl}(2)$ & $109.4(3)$ & $\mathrm{O}(2)-\mathrm{Li}(1)-\mathrm{O}(1)$ & $105.9(11)$ \\
\hline $\mathrm{N}(2)-\mathrm{Yb}(1)-\mathrm{Cl}(2)$ & $105.2(2)$ & $\mathrm{O}(4)-\mathrm{Li}(1)-\mathrm{O}(1)$ & $112.5(12)$ \\
\hline $\mathrm{N}(1)-\mathrm{Yb}(1)-\mathrm{Cl}(1)$ & $105.1(3)$ & $\mathrm{O}(2)-\mathrm{Li}(1)-\mathrm{Cl}(1)$ & $109.5(11)$ \\
\hline $\mathrm{N}(2)-\mathrm{Yb}(1)-\mathrm{Cl}(1)$ & $108.8(2)$ & $\mathrm{O}(4)-\mathrm{Li}(1)-\mathrm{Cl}(1)$ & $106.5(10)$ \\
\hline $\mathrm{Cl}(2)-\mathrm{Yb}(1)-\mathrm{Cl}(1)$ & 109.01(14) & $\mathrm{O}(1)-\mathrm{Li}(1)-\mathrm{Cl}(1)$ & $114.2(11)$ \\
\hline $\mathrm{Li}(1)-\mathrm{Cl}(1)-\mathrm{Yb}(1)$ & $175.5(6)$ & & \\
\hline
\end{tabular}

